\title{
Communication Plan As a Tool For Business Positioning
}

\section{El Plan de Comunicación Como Herramienta Orientada Al Posicionamiento Empresarial}

\author{
X. Granizo ${ }^{1}$, J. Sánchez ${ }^{1}$, B. Naula ${ }^{2}$, and D. Pazmiño ${ }^{1}$ \\ ${ }^{1}$ Escuela de Ingeniería en Marketing, Facultad de Administración de Empresas, Escuela Superior \\ Politécnica de Chimborazo, Riobamba, Ecuador \\ ${ }^{2}$ Investigador Independiente.
}

VII International Congress of

Science, Technology,

Entrepreneurship and

Innovation (SECTEI 2020)

Corresponding Author:

X. Granizo

xgranizo@espoch.edu.ec

Published: 26 August 2021

Production and Hosting by

Knowledge E

(c) X. Granizo et al. This article is distributed under the terms of the Creative Commons Attribution License, which permits unrestricted use and redistribution provided that the original author and source are credited.

\section{Abstract}

This research proposed a communication plan for the Total Home Supermarket, which is located in Riobamba city. The principal objective was to analyze the organization's communication and management with the aim of improving the management of external communication through effective strategies that achieve successful brand positioning in the local market. A qualitative and quantitative approach was applied. The types of research used were bibliographic and descriptive, supported by the inductive-deductive, analytic-synthetic and deductive methods. Primary information was collected through a survey of retail clients which examined customers' perceptions, and interviews with employees at management level. The findings showed areas of opportunity to better fulfill the organizational objectives. The development of a communication plan was proposed with effective strategies to achieve interconnected communication with the target audience; the redesign of the brand was deemed necessary since the current one is confusing due to excess information in the image. This work will promote the correct dissemination and delivery of information to the target audience through digital media, which will help the company to achieve its objectives.

Keywords: communication plan, brand, positioning, communication strategies.

\section{Resumen}

El presente trabajo propone un plan de comunicación en el Supermercado Total Home ubicada en la ciudad de Riobamba, con el objetivo de mejorar la gestión de la comunicación externa mediante estrategias efectivas que permitan lograr un posicionamiento de marca dentro del mercado riobambeño, para ello se realizó un estudio de mercado a través de encuestas aplicadas a los posibles clientes reales y potenciales de la ciudad de Riobamba, específicamente a la población económicamente activa de las parroquias urbanas para conocer la percepción del cliente hacia la marca; no obstante, para el cálculo del tamaño de la muestra se empleó el muestreo estratificado proporcional con la finalidad de evitar posibles sesgos, cuyo resultado reflejó que la empresa carece de posicionamiento; además se realizó un análisis de la situación actual de la empresa mediante las diferentes matrices con respecto a su entorno, así mismo, se realizó el análisis FODA para determinar las principales falencias presentes dentro de la organización. El desarrollo del plan de comunicación propone el direccionamiento de estrategias efectivas que logren una comunicación interconectada con el público objetivo para lo cual fue necesario el rediseño de la marca ya que la actual presenta confusión debido al 
exceso de información en una misma imagen. Se concluye que el presente trabajo fomentará la correcta difusión y entrega de información hacia el público objetivo, para lo cual se recomienda la utilización de medios digitales; de esta manera se podrá alcanzar los objetivos de la empresa.

Palabras Clave: plan de comunicación, marca, posicionamiento, estrategias de comunicación.

\section{Introducción}

El panorama de la comunicación durante los últimos años ha tenido grandes cambios y transformaciones continuos, obligando a las empresas y profesionales no solo del área comunicativa sino a investigadores de otras materias a realizar una revisión constante de esta disciplina; la forma de hacer llegar el mensaje de una manera eficaz y con el menor recurso posible a los diferentes públicos al que se dirige se ha vuelto un reto en el mundo empresarial; la comunicación en una empresa permite la difusión del producto o servicio para que las personas tengan conocimiento sobre la marca, a la vez que permite una interacción con los stakeholders.

Supermercado Total Home, es una empresa riobambeña dedicada a la comercialización de productos de consumo masivo y artículos para el hogar, cuenta con apenas 3 años en el mercado riobambeño, lo que le representa una desventaja frente a la competencia, puesto que existen tanto grandes como pequeñas empresas que llevan varios años en el mercado y por ende ya tienen posicionada su marca. No obstante, la falta de un plan de comunicación que permita potenciar la marca en un mercado tan competitivo es un factor que impide el crecimiento que la empresa desearía, pues al no contar con una correcta difusión de la marca mediante los canales más efectivos ocasiona que la competencia directa genere mayor posicionamiento.

Cabe mencionar que sin comunicación no existen ventas y por tanto la empresa 'Supermercado Total Home', sin un plan de comunicación no podrá lograr un posicionamiento de marca, dado que en ella se plantean las estrategias de comunicación necesarias y efectivas que si bien es cierto requiere de inversión pero al final generará un incremento de ventas y mayor rentabilidad. La comunicación es un proceso que permite al emisor establecer un contacto con su receptor mediante la transmisión o intercambio de información; esta acción necesaria para todo individuo permite compartir experiencias entre la persona y el mundo que lo rodea. Sin embargo, se procede a realizar un análisis de los criterios tomados de los principales autores que definen la comunicación:

Para [1], la comunicación es el conjunto de técnicas que permiten un intercambio de ideas o información con otras personas; a la vez es una realidad y un modelo cultural y 
sin ello no sería posible una vida individual y colectiva, es decir, no existe una sociedad sin comunicación. De la misma manera [2] sostienen que la comunicación es algo propio del ser humano, que si bien para muchos se considera algo inherente/innato a la misma humanidad para otros es algo natural y necesario puesto que facilita el intercambio de información mediante los distintos medios o mecanismos dentro de una sociedad. El autor [3] señala que la función de la comunicación es permitir la relación entre individuos que conviven en sociedad, y que éstos sean partícipes de las necesidades de los demás e influenciarse entre sí a través de mensajes transmitidos recíprocamente.

En la actualidad, la competencia se ha vuelto tan agresiva dentro de cualquier mercado a tal punto que obliga a las empresas plantearse estrategias de diferenciación en cuanto a su imagen e identidad, esto también se debe a la tendencia de la homogeneización de los productos y servicios, por lo que una organización debe realizar acciones de comunicación para persuadir a los públicos; estas acciones y estrategias son las que van incorporadas dentro de un plan de comunicación que facilitara a las empresas cumplir con sus objetivos [4].

Para [5] el plan de comunicación viene a ser una estrategia coordinada de acciones comunicativas que debe incluir ciertos puntos para garantizar la emisión de un mensaje efectivo hacia el público objetivo como: diagnóstico, objetivos de comunicación, público objetivo, mensaje, estrategia de comunicación, canales de comunicación, medios, acciones y tácticas que se van a realizar, evaluación de resultados y el presupuesto. Por otra parte, [6], señala que un plan de comunicación es una hoja de ruta para todos los departamentos de la empresa en el que se establece de forma clara los objetivos a alcanzar, a la vez que facilita la ordenación de las tareas desde el interior de la empresa para poder proyectar una imagen adecuada al público a la vez que utilizan de forma adecuada los recursos de la empresa.

Para los autores [2], antes de implementar un plan de comunicación es necesario tener en cuenta las etapas que lo conforman: determinar el mensaje, quien es el que va a comunicar, el destinatario, los medios y herramientas de comunicación y la obtención de resultados; este documento establece de forma clara el cómo, cuándo y de qué forma se va a comunicar la empresa con su público, con el fin de alcanzar ciertos objetivos, y que tiene como fin reforzar y ayudar al plan de marketing [7].

Dada la situación actual del mercado, en la que la competencia sobreabunda, los gestores de una organización deben identificar los atributos particulares que hacen que la imagen entregada al cliente sea diferente del resto y logre un lugar de preferencia en la mente del consumidor; sin duda, esta tarea resulta difícil debido al tiempo y ciertos elementos que requiere pero que al final resulta de vital importancia.

El posicionamiento a partir del criterio [8], es el proceso de lograr que un producto, marca, empresa o una idea se quede grabado en la mente de una persona; lo que 
conlleva a encontrar estrategias que permitan a la empresa no solo crear algo nuevo y diferente, sino manipular lo que ya está en la mente. Por otro lado, [9] sostiene que la imagen percibida por los consumidores en relación con la competencia se logra a partir de un estudio de mercado, en el cual se refleja el motivo de compra, las frecuencias de compra, las preferencias, entre otros, y de esta manera conocer el posicionamiento, las marcas que no consigan crear una posición en las mentes, se ven obligadas a reducir el precio como única vía para permanecer, por un tiempo limitado, en el mercado. Sin embargo, [10] afirma que una empresa que busca un posicionamiento positivo, debe transmitir los verdaderos atributos y beneficios de la marca de la manera más objetiva y clara posible, tratando de satisfacer la necesidad real con la autenticidad de un producto o servicio, y en cuanto a los mensajes, la comunicación y la publicidad deben ser reales. Por último, [11] señala que el posicionamiento implica reclamar una posición o reputación en un mercado, o en varios mercados, para una empresa, producto o servicio; el primer paso en el posicionamiento es identificar específicamente los mercados que se servirán; estos pueden ser locales, regionales, nacionales o incluso internacionales.

De acuerdo al criterio de estos autores, se entiende que el posicionamiento es el lugar que una marca ocupa en la mente del consumidor, por lo cual, la empresa es la responsable de otorgar una imagen propia; esta imagen propia, se construye mediante la comunicación activa de los atributos, beneficios o valores distintivos, a la audiencia objetivo, previamente seleccionados en base a las estrategias; evitando la sobreinformación, ya que hoy en día los clientes son más exigentes.

\section{Materiales y Métodos}

\subsection{Enfoque de investigación}

'El enfoque cualitativo surge al aplicar una metodología especifica orientada a captar el origen, proceso y naturaleza de estos significados que brotan de la interacción simbólica entre individuos' [12]. Este enfoque se lo utilizó al momento de realizar el análisis situacional de la empresa, mediante un trabajo de campo, utilizando técnicas e instrumentos de investigación a cierto porcentaje de la población urbana.

En lo que se refiere al enfoque cuantitativo 'Utiliza la recolección y análisis de datos para contestar preguntas de investigación y probar las hipótesis establecidas previamente y confía en la medición numérica, el conteo y en el uso de la estadística para establecer con exactitud los patrones en una población' [13]. El mismo que se utilizó al momento de la recopilación de información mediante la encuesta aplicada, mediante la cual se pudo determinar el nivel de posicionamiento que posee el Supermercado Total Home. 


\subsection{Nivel de Investigación}

‘El nivel de estudio de la presente investigación es exploratorio, "Este tipo de estudio ayuda a dividir un problema muy grande y llegar a unos sub- problemas más precisos hasta la forma de expresar las hipótesis; es utilizada para generar el criterio y dar prioridad a algunos problemas"' [14]. El tema planteado requirió de un estudio que aún no ha sido abordado dentro de la empresa, por lo cual se buscó explorar y examinar la situación actual de la misma y recolectar los datos necesarios.

En lo referente al nivel descriptivo 'Comprende la descripción, registro, análisis e interpretación de la naturaleza actual, composición o procesos de fenómenos' [15]. Se efectuó al momento de describir los hechos tal y cómo son observados, para el cual se acudió a la utilización de técnicas como la observación directa y la revisión bibliográfica y posterior a ello se pudo realizar comparaciones y comprobaciones de resultados.

\subsection{Diseño de investigación}

El diseño de la presente investigación es de tipo no experimental ya que no se requirió ningún tipo de experimento o utilización de equipos biométricos para su estudio; es decir que no se manipularon las variables del estudio. El estudio realizado es transversal debido a que se basa en la observación del entorno real. Una vez elegido el objetivo del estudio, se comparan al mismo tiempo determinadas características o situaciones dadas en la empresa.

\subsection{Población y muestra}

Para la siguiente investigación se realizó un análisis de la población económicamente activa (PEA) de la ciudad de Riobamba urbano, que según datos del Instituto Nacional de Estadísticas y Censos (INEC) son 144.683 personas, los cuales constituyen el universo de estudio.

Sin embargo, para esta investigación se aplicó una encuesta piloto que permita determinar el porcentaje de la probabilidad de ocurrencia (p) y la probabilidad de que el evento no ocurra (q), así mismo para medir el nivel de posicionamiento que tiene la empresa actualmente.

El cálculo de la muestra se realiza con el fin de conocer el número exacto de las personas sobre los cuales se harán el estudio de campo, los mismos que deben ser datos representativos de la población. Para ello, se utilizará la fórmula para calcular poblaciones finitas, puesto que se conoce el número del universo a estudiar:

$$
n=\frac{Z^{2} P Q N}{E^{2}(N-1)+Z^{2} P Q},
$$


dónde: $n$ : Tamaño de la muestra; $N$ : Tamaño de la población; $Z$ : Niveles de confianza (1,96); E: Error muestral (0,05); P: Probabilidad de ocurrencia; Q: Probabilidad de que el evento no suceda.

Al aplicar la formula, se obtiene el siguiente resultado:

$$
\begin{gathered}
n=\frac{(1.96)^{2}(0.2)(0.8)(144.683)}{(0.05)^{2}(144.683-1)+(1.96)^{2}(0.2)(0.8)}, \\
n=\frac{88930.27}{363}, \\
n=245 \text { encuestas. }
\end{gathered}
$$

Para la recolección de información realizada en el estudio de campo se aplicó el muestreo probabilístico (estratificado proporcional); es decir, el número de encuestas realizada en cada una de las 5 parroquias urbanas de la ciudad de Riobamba se determinó de acuerdo al número de habitantes (observar Tabla 1) con la finalidad de evitar posibles sesgos, a la vez conocer las parroquias en las que menos o más participación de mercado tiene la empresa.

\section{Table 1}

Número de encuestas por parroquias de la ciudad de Riobamba.

\begin{tabular}{l|l|l|l}
\hline Parroquias & PEA & \% & \\
\hline Lizarzaburu & 50,321 & $35 \%$ & 86 \\
\hline Maldonado & 31,856 & $22 \%$ & 54 \\
\hline Velasco & 34,814 & $24 \%$ & 58 \\
\hline Veloz & 21,694 & $15 \%$ & 37 \\
\hline Yaruquíes & 5,998 & $4 \%$ & 10 \\
\hline Total & $\mathbf{1 4 4 , 6 8 3}$ & $\mathbf{1 0 0 \%}$ & $\mathbf{2 4 5}$
\end{tabular}

\subsection{Métodos, técnicas e instrumentos de investigación}

'El método inductivo es un proceso en el que, a partir del estudio de casos particulares, se obtienen conclusiones o leyes universales que explican o relacionan los fenómenos estudiados' [15]. Este método se utilizó en la observación del fenómeno de estudio analizando la marca actual de la empresa y a partir de ello llegar a una conclusión que permita determinar la solución más efectiva.

'El método deductivo consiste en obtener conclusiones particulares a partir de una ley universal' [15]. Este método se lo utilizó para la descripción detallada de cada una de las variables que se encuentran inmersas en el tema de estudio, variable independiente (plan de comunicación), variable dependiente (posicionamiento de la empresa). 
El método analítico fue utilizado al momento de estudiar los aspectos particulares de la situación actual de la empresa con respecto a la comunicación. El método sintético se utilizó al momento de dar las interpretaciones necesarias a la información obtenida del estudio mercado.

\subsection{Técnicas de recopilación de información}

Las técnicas de recopilación de información utilizadas en la presente investigación fueron la observación directa, mediante las visitas realizadas a la empresa para recabar información directa sobre la interacción del establecimiento con los clientes y la relación existente con los mismos. Mediante la aplicación de una entrevista realizada al gerente de la empresa se obtuvo información importante sobre las actividades de dirección de la misma y las funciones que realiza el personal. La encuesta aplicada a la población de estudio permitió la recopilación de información relevante, mediante el cual se pudo determinar el perfil del consumidor, conocer su opinión, preferencias, expectativas y sobre todo medir el posicionamiento que tiene la empresa con respecto a la competencia.

\section{Resultados y Discusión}

El desarrollo de la presente investigación se centra en crear una imagen de marca que genere oportunidades de negocio; para lo cual se tomó en cuenta los principales hallazgos del estudio realizado, el cual permitió determinar que el $80 \%$ de la población riobambeña desconoce la existencia de dicha empresa, dentro del $20 \%$ de personas que dijeron saber sobre la existencia de la empresa, afirmaron conocerla por casualidad, por recomendación de amigos y familiares, mas no por haber visto publicidad sobre la empresa en algún medio. El 49\% de los encuestados recomiendan que la empresa debe invertir en publicidad, siendo las redes sociales una de las herramientas que facilitan la difusión de la marca, razón por lo que el 74\% de encuestados sugiere la utilización de este medio. Estos, entre otros hallazgos contribuyeron a que la empresa plantee estrategias efectivas de comunicación que permitan una participación de mercado, siendo un referente en cuanto a la actividad del comercio minorista se refiera.

Como se muestra en la Tabla 2, apenas el $20 \%$ de la población encuestada afirmó conocer la marca de la empresa Total Home frente al $80 \%$ que afirmó no reconocerla, resultado que evidencia que el supermercado cuenta con un bajo nivel de posicionamiento en el mercado local.

La Tabla 3 evidencia el medio a través del cual la empresa ha logrado posicionarse dentro del $20 \%$ de la población encuestada, que asciende a 49 personas; el 59\% de este total afirmó conocer la empresa por casualidad, el 39\% por recomendación de 
Table 2

Reconocimiento de la marca.

Lizarzaburu
Maldonado
Velasco
Veloz
Yaruquíes

Sí
14
15
6
5
9
49

$\%$
$16 \%$
$28 \%$
$10 \%$
$14 \%$
$90 \%$
$20 \%$

No.
72
39
52
32
1
196

$\%$
$84 \%$
$72 \%$
$90 \%$
$87 \%$
$10 \%$
$80 \%$

Total
86
54
58
37
10
245

Table 3

Cómo se enteró de la existencia del supermercado.

\begin{tabular}{l|l|l|} 
& Recomendación \\
\hline Lizarzaburu & 6 \\
\hline Maldonado & 5 \\
\hline Velasco & 3 \\
\hline Veloz & 2 \\
\hline Yaruquíes & 3 \\
& 19 \\
\hline
\end{tabular}

\begin{tabular}{l}
$\%$ \\
$43 \%$ \\
$33 \%$ \\
$50 \%$ \\
$40 \%$ \\
$33 \%$ \\
$39 \%$ \\
\hline
\end{tabular}

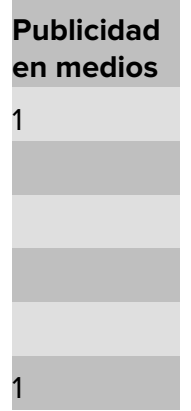

\begin{tabular}{|l|l|}
\hline$\%$ & Casualidad \\
\hline $7 \%$ & 7 \\
& 10 \\
& 3 \\
& 3 \\
\hline & 6 \\
\hline & 29 \\
\hline
\end{tabular}

\begin{tabular}{|l|l|}
\hline$\%$ & Total \\
\hline $50 \%$ & 14 \\
\hline $67 \%$ & 15 \\
\hline $50 \%$ & 6 \\
$60 \%$ & 5 \\
\hline $67 \%$ & 9 \\
\hline $59 \%$ & 49 \\
\hline
\end{tabular}

terceros, y apenas el $2 \%$ del total señaló conocer a la empresa a través de la publicidad en medios que realiza la misma. Resultado que evidencia que la empresa no emplea ningún medio de comunicación que permita difundir su marca.

\section{Table 4}

Aspectos a considerar para la mejora del S.T.H.

\begin{tabular}{l|l|} 
& $\begin{array}{l}\text { Mejorar la } \\
\text { marca }\end{array}$ \\
\hline Lizarzaburu & 4 \\
\hline Maldonado & 2 \\
\hline Velasco & 1 \\
\hline Veloz & 2 \\
\hline Yaruquíes & \\
\hline & 9
\end{tabular}

\begin{tabular}{|l|l|}
\hline$\%$ & $\begin{array}{l}\text { Incrementar } \\
\text { publicidad }\end{array}$ \\
\hline $29 \%$ & 7 \\
$13 \%$ & 6 \\
$17 \%$ & 3 \\
\hline $40 \%$ & 3 \\
\hline $18 \%$ & 5 \\
\hline
\end{tabular}

$\%$
$50 \%$
$40 \%$
$50 \%$
$60 \%$
$56 \%$
$49 \%$

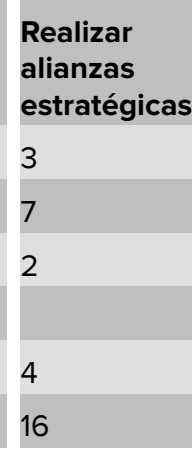

\begin{tabular}{|l|l|}
\hline$\%$ & Total \\
\hline $21 \%$ & 14 \\
\hline $47 \%$ & 15 \\
\hline $33 \%$ & 6 \\
\hline $44 \%$ & 5 \\
\hline $33 \%$ & 49 \\
\hline
\end{tabular}

En lo que respecta a las 49 personas que afirmaron conocer la empresa, el $49 \%$ de este total sugiere a la empresa la inversión en publicidad, recomendación que presenta un alto porcentaje en casi todas las parroquias, el 33\% de los encuestados sugiere el establecimiento de alianzas estratégicas que permitan dar a conocer a la empresa al 
público, ejemplo de estas son los auspicios a eventos deportivos locales que permiten la difusión de la marca y contribuyen con el posicionamiento de mercado.

Table 5

Aceptación de la marca.

\begin{tabular}{l|l|l|l|l|l|} 
& No. & $\%$ & Si & $\%$ & Total \\
\hline Lizarzaburu & 6 & $7 \%$ & 80 & $93 \%$ & 86 \\
\hline Maldonado & 5 & $9 \%$ & 49 & $91 \%$ & 54 \\
\hline Velasco & 4 & $7 \%$ & 54 & $93 \%$ & 58 \\
\hline Veloz & 6 & $16 \%$ & 31 & $84 \%$ & 37 \\
\hline Yaruquíes & 4 & $40 \%$ & 6 & $60 \%$ & 10 \\
& 25 & $10 \%$ & 220 & $90 \%$ & 245
\end{tabular}

Acorde a los resultados expuestos en la Tabla 5, el 90\% de las personas encuestadas sostienen que si les gustaría recibir más información sobre la empresa, lo que representa una excelente oportunidad para establecer estrategias de comunicación que contribuyan con el posicionamiento de la empresa. Apenas el 10\% de encuestados dispersos en todas las parroquias no se encuentran interesados en recibir información sobre la empresa.

Table 6

Medios de información con mayor aceptación.

\begin{tabular}{|l|l|l|l|l|l|l|l|} 
& $\begin{array}{l}\text { Es sociales, } \\
\text { inas web }\end{array}$ & \% & $\begin{array}{l}\text { Medios } \\
\text { masivos (tv, } \\
\text { radio) }\end{array}$ & $\%$ & $\begin{array}{l}\text { Medios } \\
\text { impresos }\end{array}$ & \% & Total \\
\hline Lizarzaburu & 61 & $71 \%$ & 12 & $14 \%$ & 13 & $15 \%$ & 86 \\
\hline Maldonado & 38 & $70 \%$ & 9 & $17 \%$ & 7 & $13 \%$ & 54 \\
\hline Velasco & 42 & $73 \%$ & 6 & $10 \%$ & 10 & $17 \%$ & 58 \\
\hline Veloz & 35 & $94 \%$ & 1 & $3 \%$ & 1 & $3 \%$ & 37 \\
\hline Yaruquíes & 7 & $70 \%$ & & & 3 & $30 \%$ & 10 \\
& 183 & $75 \%$ & 28 & $11 \%$ & 34 & $14 \%$ & 245 \\
\hline
\end{tabular}

Acorde a la tendencia del uso de las tecnologías de la información y los resultados que evidencia la Tabla 6, el medio de comunicación por el cual las personas afirman desearían obtener información sobre la empresa es a través de los medios digitales, específicamente las redes sociales como lo afirma el $75 \%$ de los encuestados, medio preferido por la población gracias a la accesibilidad del mismo; el 14\% de encuestados señala a los medios impresos como su medio predilecto para recibir información y apenas un $11 \%$ seleccionó los medios masivos como la televisión y radio como el medio ideal para recibir información sobre la empresa. 


\subsection{Análisis macro-ambiental}

Con la finalidad de diseñar e implementar estrategias de comunicación competitivas en el mercado se realizó un análisis del sector comercial en el que se encuentra inmersa la empresa, mediante el análisis (PEST) de los factores políticos, económicos, sociales y tecnológicos y la matriz de factores externos MEFE se analizó el macro-entorno y mediante el análisis de las 5 Fuerzas de Porter se procedió con el análisis del microentorno.

Para el desarrollo de la matriz PEST se consideraron los factores políticos, económicos, sociales, culturales y tecnológicos; con el fin de establecer aquellas variables que afectan a la empresa y también aquellos factores que con el tiempo podrían ir cambiando para el desarrollo óptimo de la organización.

Mediante esta herramienta se puede reconocer todos aquellos factores externos sobre los cuales la empresa tenga influencia y posterior a ello aminorar los efectos de las amenazas, los cuales son muy útil al momento de realizar planes de negocios, marketing y plan estratégico; esta herramienta resulta adaptable a cualquier empresa consolidada, sea de pequeña como grande. [16]

A partir del análisis a nivel macro establecido en la matriz se puede identificar que existen ciertos factores menos favorecedores para la empresa, se destacan en el factor político: la inestabilidad, las leyes laborales; en el factor económico: la situación actual del país y la inflación, puesto que al disminuir el ingreso de los clientes actuales y potenciales afectan directamente su poder adquisitivo y por ende el desempeño de la empresa. En cuanto a los factores que favorecen a la empresa están los factores tecnológicos, considerados como un medio efectivo para penetrar el mercado objetivo y lograr el desarrollo de la empresa, así mismo el crecimiento poblacional y el estilo de vida de la población riobambeña.

\subsection{Matriz del Perfil Competitivo (MPC)}

La finalidad de la Matriz del Perfil Competitivo es determinar los factores críticos claves del éxito mediante una comparación de la empresa frente a sus rivales, de esta manera conocer las fortalezas y debilidades relativas en función de su competencia [17].

Para la siguiente matriz se tomó en cuenta 2 de los supermercados de la ciudad (Tía, Ahí Es) debido a que se encuentran ubicados dentro de la misma zona comercial y que por tanto se consideran sus principales competidores.

Mediante la matriz del perfil competitivo se pudo determinar que la empresa más fuerte en términos competitivos es 'Almacenes Tía' con un puntaje casi perfecto $(4,36)$ debido a la participación de mercado, la innovación constante y el posicionamiento de marca del que goza la empresa; por su parte la empresa 'Supermercado Total Home' 


\begin{tabular}{|c|c|c|c|c|c|c|}
\hline \multirow{2}{*}{$\begin{array}{l}\text { Factores } \\
\text { claves Del } \\
\text { entorno }\end{array}$} & \multirow[b]{2}{*}{ Variables } & \multicolumn{5}{|l|}{ Impacto } \\
\hline & & Muy negativo & Negativo & Equilibrado & Positivo & $\begin{array}{l}\text { Muy } \\
\text { positivo }\end{array}$ \\
\hline \multirow{4}{*}{ Político } & Inestabilidad política & $x$ & & & & \\
\hline & Política de impuestos & & & & $x$ & \\
\hline & Ley laboral & & $x$ & & & \\
\hline & $\begin{array}{l}\text { Relación entre gobierno y } \\
\text { ciudadano }\end{array}$ & & & $x$ & & \\
\hline \multirow{6}{*}{ Económico } & Evolución del PIB & & & & $x$ & \\
\hline & $\begin{array}{l}\text { Situación económica del } \\
\text { país }\end{array}$ & $x$ & & & & \\
\hline & $\begin{array}{l}\text { Poder adquisitivo de los } \\
\text { clientes }\end{array}$ & & & & & $x$ \\
\hline & Inflación & $x$ & & & & \\
\hline & Tipo de cambio (\$) & & & & $x$ & \\
\hline & Incremento del riesgo país & & $x$ & & & \\
\hline \multirow{5}{*}{$\begin{array}{l}\text { Socio- } \\
\text { cultural }\end{array}$} & Corrupción & $x$ & & & & \\
\hline & Hábitos de consumo & & & & & $x$ \\
\hline & Clase social & & & & $x$ & \\
\hline & Crecimiento poblacional & & & & & $x$ \\
\hline & Estilo de vida & & & & $x$ & \\
\hline \multirow{3}{*}{ Tecnológico } & $\begin{array}{l}\text { Inversión en Investigación y } \\
\text { desarrollo de nuevos } \\
\text { productos }\end{array}$ & & & & $x$ & \\
\hline & $\begin{array}{l}\text { Acceso a nuevas } \\
\text { tecnologías }\end{array}$ & & & & $x$ & \\
\hline & Tecnología de compra & & & & $x$ & \\
\hline
\end{tabular}

Figure 1

Matriz PEST.

Table 7

Matriz del Perfil Competitivo.

\begin{tabular}{|c|c|c|c|c|c|c|c|}
\hline \multirow[b]{2}{*}{ Factores clave } & \multirow[b]{2}{*}{ Peso } & \multicolumn{2}{|c|}{ Total home } & \multicolumn{2}{|c|}{ Almacenes tía } & \multicolumn{2}{|c|}{ Ahí es! } \\
\hline & & Valor & Ponderado & Valor & Ponderado & Valor & Ponderado \\
\hline 1 Participación & 0,1 & 1 & 0,1 & 4 & 0,4 & 3 & 0,3 \\
\hline 2 de mercado & 0,12 & 4 & 0,48 & 4 & 0,48 & 4 & 0,48 \\
\hline 3 Precios & 0,12 & 3 & 0,36 & 3 & 0,36 & 3 & 0,36 \\
\hline 4 competitivos & 0,1 & 1 & 0,1 & 4 & 0,4 & 2 & 0,2 \\
\hline 5 Variedad de & 0,18 & 3 & 0,54 & 4 & 0,72 & 3 & 0,54 \\
\hline 6 los productos & 0,2 & 3 & 0,6 & 4 & 0,8 & 3 & 0,6 \\
\hline 7 Publicidad & 0,12 & 2 & 0,30 & 5 & 0,6 & 4 & 0,48 \\
\hline $\begin{array}{l}8 \text { Marca Innovación } \\
\text { Comunicación }\end{array}$ & 0,15 & 2 & 2,48 & 4 & 0,6 & 4 & 0,6 \\
\hline $\begin{array}{l}\text { externa Imagen } \\
\text { corporativa }\end{array}$ & 1 & & & & 4,36 & & 3,56 \\
\hline TOTAL & & & & & & & \\
\hline
\end{tabular}


carece de estos factores que ayuden en su crecimiento exitoso dentro de la industria, pero maneja precios competitivos y variedad de productos al igual que sus principales competidores. El peso asignado a cada factor clave, se definió mediante criterios de la observación directa y también de acuerdo al estudio de mercado realizado, mediante el cual se pudo determinar que 'Almacenes Tía' obtuvo el mayor porcentaje de participación de mercado, equivalente al 60\%, mientras que el supermercado 'Ahí Es' cuenta con el $20 \%$ de participación.

\subsection{Análisis micro-ambiental}

El modelo de las 5 fuerzas de Michael Porter planteado por Michael Porter permite medir la competencia e identificar las oportunidades de la industria a partir de 4 fuerzas: amenaza de competidores, amenaza de nuevos productos, poder de negociación con los proveedores y poder de negociación con los clientes; posterior a ello medir otra fuerza que es la rivalidad entre competidores [18].

La siguiente matriz de cinco fuerzas de Porter se emplea con el fin de identificar la rivalidad de la empresa con la competencia dentro del mercado riobambeño y determinar de la manera más eficaz las fuerzas competitivas a desarrollar para lograr un posicionamiento y permanencia en el mercado.

La empresa 'Supermercado Total Home' al ingresar a la industria de los supermercados tuvo como barrera de entrada la falta de experiencia; sin embargo, los productos de calidad y los precios competitivos que manejan permitieron mantener una rentabilidad al margen.

En cuanto a la rivalidad de la competencia maneja un panorama hostil, debido a que está rodeado tanto de las grandes marcas como de negocios familiares en una zona muy comercial (La Condamine), las empresas de esta industria cuentan con varios años en el mercado y cuentan con un reconocimiento de marca y clientes fieles por lo que el supermercado Total Home requiere de gran inversión en publicidad, ya que la imagen, credibilidad, seriedad y fiabilidad que tiene actualmente es muy débil debido a la forma de actuar de la empresa y de las características de los productos que oferta; es así que la empresa no han logrado que el comprador relacione el producto con la marca.

En cuanto al poder de negociación con los clientes es medianamente favorable ya que los compradores que acuden al lugar adquieren sus productos de acuerdo al precio que está marcado en cada uno de ellos y sobre todo va acorde a la del mercado. Los productos sustitutos se encuentran en una escala hostil, ya que se los puede encontrar en lugares de fácil acceso como los mercados y tiendas de barrio. Es así que la empresa para protegerse de estas amenazas debe buscar diversificar sus productos tradicionales. 


\begin{tabular}{|c|c|c|c|c|c|c|c|}
\hline \multicolumn{8}{|c|}{ Matriz Cinco Fuerzas de Michael Porter } \\
\hline \multicolumn{8}{|c|}{$\begin{array}{l}\text { A continuación, marque con una } X \text { en las siguientes casillas según el estado actual de su empresa. } \\
\text { Valore su perfil competitivo en la escala Hostil-Favorable. }\end{array}$} \\
\hline \multirow{2}{*}{ Perfil competitivo } & \multirow{2}{*}{ Hostil } & \multicolumn{5}{|c|}{ Valoración } & \multirow{2}{*}{ Favorable } \\
\hline & & 1 & 2 & 3 & 4 & 5 & \\
\hline \multicolumn{8}{|l|}{ Rivalidad empresas del sector } \\
\hline Crecimiento de competidores & Lento & & & & & $x$ & Rápido \\
\hline Número de competidores & Muchos & & & & $x$ & & Pocos \\
\hline Publicidad desleal & $\mathrm{Si}$ & & $\mathrm{x}$ & & & & No \\
\hline Rentabilidad media del sector & Baja & & & $\mathrm{x}$ & & & Alta \\
\hline Guerra de precios & Alta & & & $\mathrm{x}$ & & & Baja \\
\hline \multicolumn{8}{|l|}{ Barreras de entrada } \\
\hline Economía de escala & No & & $\mathrm{x}$ & & & & $\mathrm{Si}$ \\
\hline Diferenciación & No & & $\mathrm{x}$ & & & & $\mathrm{Si}$ \\
\hline Requerimiento de capital & Fácil & & & $x$ & & & Difícil \\
\hline Experiencia & Escasa & & $\mathrm{x}$ & & & & Suficiente \\
\hline $\begin{array}{l}\text { Acceso a los canales de } \\
\text { distribución }\end{array}$ & Fácil & & $x$ & & & & Difícil \\
\hline Identificación de marca & Fácil & & & & & $x$ & Difícil \\
\hline \multicolumn{8}{|l|}{ Poder de los clientes } \\
\hline Número de clientes & Pocos & & & & $\mathrm{x}$ & & Muchos \\
\hline Posibilidad de integración & Grande & & & $x$ & & & Pequeña \\
\hline Exigencia de los clientes & Alta & & & & $x$ & & Baja \\
\hline Relación con los clientes & Pésima & & $\mathrm{x}$ & & & & Excelente \\
\hline Calidad en los productos & Baja & & & & $\mathrm{x}$ & & Alta \\
\hline \multicolumn{8}{|l|}{ Productos sustitutos } \\
\hline $\begin{array}{l}\text { Disponibilidad de productos } \\
\text { sustitutos }\end{array}$ & Grande & & $x$ & & & & Pequeña \\
\hline $\begin{array}{l}\text { Precio productos sustitutos vs } \\
\text { analizado }\end{array}$ & Bajo & & & & $x$ & & Alto \\
\hline \multicolumn{8}{|l|}{ Poder de proveedores } \\
\hline Número de proveedores & Bajo & & & & $x$ & & Alto \\
\hline Posibilidad de integración & Alto & & & $x$ & & & Bajo \\
\hline Relación con los proveedores & Pésima & & & & $x$ & & Excelente \\
\hline Precios atractivos & Altos & & & & $\mathrm{x}$ & & Bajos \\
\hline
\end{tabular}

Figure 2

Matriz de las 5 fuerzas de Porter.

El poder de negociación con los proveedores es medianamente favorable, las grandes empresas buscan nichos de mercados como por ejemplo los supermercados para ubicar sus productos dentro de las perchas de estos lugares por lo que a la empresa le facilitan los procesos.

\subsection{Matriz de Evaluación de los factores externos (MEFE)}

La matriz es una herramienta de diagnóstico que permite realizar un estudio de campo, permitiendo identificar y evaluar los diferentes factores externos que pueden influir con el crecimiento y expansión de una marca, dentro del instrumento facilita la formulación de diversas estrategias que son capaces de aprovechar las oportunidades y minimizar los peligros externos [19]. 
Una evaluación completa del entorno permite a la empresa generar una valoración al desempeño del mismo; es por ello la importancia de considerar las amenazas y oportunidades presentes en el medio, aprovechar al máximo las fortalezas de la empresa puede evitar un rezago frente a la competencia; para la realización de esta matriz se tomó en cuenta los factores críticos que generen oportunidades y las amenazas existentes o futuras que impidan el desarrollo empresarial.

\section{Table 8}

\section{Matriz MEFE.}

\begin{tabular}{l|l|l|l}
$\begin{array}{l}\text { Matriz de Evaluacion de los Factores } \\
\text { Externos Factores críticos }\end{array}$ & Peso & Calificación & Puntuación \\
$\begin{array}{l}\text { OPORTUNIDADES } \\
\begin{array}{l}\text { Atraer clientes y buscar nuevos nichos de } \\
\text { mercado }\end{array}\end{array}$ & 0,12 & 2 & 0,24 \\
$\begin{array}{l}\text { Cubrir la demanda del mercado } \\
\text { Buscar nuevos canales de venta y alianzas } \\
\text { estratégicas }\end{array}$ & 0,1 & 2 & 0,2 \\
\hline $\begin{array}{l}\text { Tecnología actualizada } \\
\text { Diversificación de la línea de productos }\end{array}$ & 0,1 & 2 & 0,18 \\
\hline $\begin{array}{l}\text { AMENAZAS } \\
\text { Incumplimiento de tareas y } \\
\text { responsabilidades de los miembros y }\end{array}$ & 0,03 & 1 & 0,24 \\
\hline $\begin{array}{l}\text { proveedores. Nula participación de mercado } \\
\text { Pérdida financiera, fracaso de la empresa }\end{array}$ & 0,1 & 1 & 0,1 \\
\hline $\begin{array}{l}\text { Desconocimiento de clientes reales y } \\
\text { potenciales. }\end{array}$ & 0,08 & 3 & 0,90 \\
\hline $\begin{array}{l}\text { Pérdida de clientes } \\
\text { Total }\end{array}$ & 0,12 & 2 & 0,2 \\
\hline
\end{tabular}

Como se pudo observar, acorde a los resultados que muestra la Tabla 8, el entorno genera una serie de oportunidades que la empresa puede aprovechar para contrarrestar las amenazas; sin embargo, de acuerdo a la evaluación de los factores externos, el valor de ponderado total es de 2,23 por debajo del promedio recomendado $(2,5)$; lo que nos indica que la empresa no está respondiendo de manera excelente a las oportunidades para contrarrestar las amenazas existentes en su actividad económica; como se pudo observar, los factores más importantes que afectan a la empresa son la 'pérdida de clientes', 'fidelización' y la búsqueda de 'nuevos nichos de mercado' como lo señala el peso de 0,12. La empresa no cuenta con estrategias que capitalicen muy bien esta oportunidad pues estos factores responden a un $2 / 4$ como lo señala la calificación; es decir que las estrategias que emplea la empresa son nulas para lograr una participación en el mercado.

A partir de los análisis presentados se plantean estrategias de comunicación mediante los medios más utilizados en la actualidad, los mismos que están orientados a 
Table 9

FODA estratégico.

\begin{tabular}{|c|c|c|c|}
\hline \multirow{2}{*}{\multicolumn{2}{|c|}{ FODA }} & ORTALEZAS & \multirow[b]{2}{*}{$\begin{array}{l}\text { DEBILIDADES } \\
\text { D1. Mala estructura organizativa } \\
\text { D2. Poca publicidad de marca en los } \\
\text { medios } \\
\text { D3. Falta de capacitación al personal } \\
\text { D4. Carencias de estudio de mercado } \\
\text { D5. Déficit de cajeros para la atención en } \\
\text { días y horas pico. }\end{array}$} \\
\hline & & $\begin{array}{l}\text { 1. Precios competitivos } \\
=2 \text {. Productos de gran variedad y de } \\
\text { econocidas marcas. } \\
=3 \text {. Buena ubicación de la empresa } \\
\text { 4. Infraestructura propia } \\
\text { =5. Adaptación continúa hacia la } \\
\text { exigencia de los consumidores. }\end{array}$ & \\
\hline $\begin{array}{l}\text { 岀 } \\
\text { 是 } \\
\text { 竞 } \\
\text { 름 }\end{array}$ & $\begin{array}{l}\text { 01. Atraer clientes } \\
\text { y buscar nuevos } \\
\text { nichos de } \\
\text { mercado } \\
\text { 0 2. Cubrir la } \\
\text { demanda del } \\
\text { mercado } \\
\text { O3. Nuevos } \\
\text { canales de venta y } \\
\text { alianzas } \\
\text { estratégicas } \\
\text { O4. Tecnología } \\
\text { actualizada } \\
\text { 05. Participación } \\
\text { en la industria de } \\
\text { nuevos productos. }\end{array}$ & $\begin{array}{l}\text { F4 - 01 Aprovechar la ubicación de } \\
\text { la empresa e invertir en publicidad } \\
\text { externa para captar la atención de } \\
\text { nuevos clientes. } \\
\text { F1 - O3: Crear nueva línea de } \\
\text { productos con marca propia } \\
\text { manteniendo los precios } \\
\text { competitivos, mediante alianzas } \\
\text { estratégicas. } \\
\text { F2 - 02: Aprovechar a variedad de } \\
\text { los productos para incentivar } \\
\text { clientes insatisfechos. }\end{array}$ & $\begin{array}{l}\text { D2 - 01: Rediseñar el organigrama de la } \\
\text { empresa y delegar funciones específicas a } \\
\text { los miembros para la discusión de la } \\
\text { marca y llegar a más personas. } \\
\text { D2 - O3: Ubicar soportes visuales con } \\
\text { información sobre las nuevas líneas de } \\
\text { productos, en las principales zonas } \\
\text { urbanas de la ciudad. } \\
\text { D5 - 04: Incrementar el número de cajas, } \\
\text { otorgando prioridad para personas } \\
\text { embarazadas y capacidad especial, de } \\
\text { esta manera fidelizar al cliente. }\end{array}$ \\
\hline 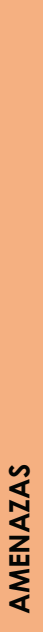 & $\begin{array}{l}\text { A1. } \\
\text { Incumplimiento } \\
\text { de tareas y } \\
\text { responsabilidades } \\
\text { de los miembros y } \\
\text { proveedores. } \\
\text { A2. Perder } \\
\text { participación en el } \\
\text { mercado } \\
\text { A3. Pérdida } \\
\text { financiera, fracaso } \\
\text { de la empresa } \\
\text { A4. } \\
\text { Desconocimiento } \\
\text { de clientes reales y } \\
\text { potenciales. } \\
\text { A5. Pérdida de } \\
\text { clientes }\end{array}$ & $\begin{array}{l}\text { F5 A5: Mantener un } \\
\text { cumplimiento de las exigencias y } \\
\text { responsabilidades para evitar una } \\
\text { pérdida de clientes. } \\
\text { F2 - A2: Promocionar las ventajas } \\
\text { de los productos con respecto a la } \\
\text { para ganar participación de } \\
\text { mercado } \\
\text { F1 - A1: Buscar soluciones para } \\
\text { evitar posibles conflictos entre } \\
\text { departamentos }\end{array}$ & $\begin{array}{l}\text { D4 - A4: F3 - A4: Realizar un estudio de } \\
\text { mercado para conocer el } \\
\text { posicionamiento actual que tiene la } \\
\text { empresa y conocer a los posibles clientes } \\
\text { reales y potenciales. } \\
\text { D1 - A3: Con los precios competitivos que } \\
\text { trabaja la empresa lograra una } \\
\text { participación de mercado. } \\
\text { F2 - D5: Crear promociones en productos } \\
\text { seleccionados para captar la atención de } \\
\text { clientes reales y potenciales }\end{array}$ \\
\hline
\end{tabular}

lograr un posicionamiento en el mercado mediante objetivos AIDA (atención, interés, deseo y acción). Cabe mencionar que el modelo 478 AIDA es un clásico en el mundo del marketing y las ventas, el mismo que describe las fases que sigue un cliente en el proceso de decisión de compra [20].

\subsection{Estrategias prpuestas}




\subsubsection{Medios de comunicación masiva ATL: Spots publicitarios en tele- visión y radio}

Table 10

Estrategia 1. Cuña publicitaria en radio.

\begin{tabular}{|c|c|c|c|c|}
\hline Descripción & \multicolumn{4}{|c|}{$\begin{array}{l}\text { La radio es un buen canal de marketing promocional y una de las estrategias más } \\
\text { utilizadas para difundir cualquier información gracias al gran impacto que genera en } \\
\text { el público. }\end{array}$} \\
\hline Objetivo & \multicolumn{4}{|c|}{$\begin{array}{l}\text { Acceder a un público más amplio y lograr que la audiencia se incline hacia la compra } \\
\text { del producto en nuestra empresa. }\end{array}$} \\
\hline Estrategia & \multicolumn{4}{|c|}{ Establecer cuña radial en horas pico $14 \mathrm{HOO}-17 \mathrm{HOO}$. } \\
\hline $\begin{array}{l}\text { Audiencia a } \\
\text { impactar }\end{array}$ & \multicolumn{4}{|c|}{ Clientes reales y potenciales. } \\
\hline Responsable & \multicolumn{4}{|c|}{ Departamento de Marketing. } \\
\hline Táctica & \multicolumn{4}{|c|}{$\begin{array}{l}\text { - Crear un spot publicitario en radio con una duración de } 30 \text { segundos. } \\
\text { - Utilizar el efecto 'AIDA' en la presentación. }\end{array}$} \\
\hline Frecuencia & \multicolumn{4}{|c|}{2 veces por día, durante 3 meses. } \\
\hline Medio & \multicolumn{4}{|c|}{ Radio: Emisora 'Bonita FM' } \\
\hline \multirow[t]{2}{*}{ Costo } & Diario & Semanal & Mensual & Trimestral \\
\hline & $\$ 12,50$ & $\$ 25,00$ & $\$ 200,00$ & $\$ 600,00$ \\
\hline
\end{tabular}

\section{Table 11}

Estrategia 2. Spot publicitario TV.

\begin{tabular}{|c|c|c|c|}
\hline Descripción & \multicolumn{3}{|c|}{$\begin{array}{l}\text { La televisión es un medio masivo que logra una difusión mucho más efectiva } \\
\text { que la radio, puesta que genera un mayor impacto a través de los soportes } \\
\text { televisivos. }\end{array}$} \\
\hline Objetivo & \multicolumn{3}{|c|}{ Difundir la marca y el consumo de los productos que oferta la empresa. } \\
\hline Estrategia & \multicolumn{3}{|c|}{ Publicitar la marca mediante televisión local: canal 13 TVSultana, horarios AAA. } \\
\hline Audiencia a impactar & \multicolumn{3}{|c|}{ Clientes reales y potenciales. } \\
\hline Responsable & \multicolumn{3}{|c|}{ Departamento de Marketing. } \\
\hline Táctica & \multicolumn{3}{|c|}{$\begin{array}{l}\text { - Diseñar un spot publicitario para televisión con una duración de } 30 . \\
\text { - Utilizar el efecto 'AIDA' durante el spot. }\end{array}$} \\
\hline Frecuencia & \multicolumn{3}{|c|}{ Mensual: Dos veces por semana Horarios: 08 h00 a 09h00. } \\
\hline Medio & \multicolumn{3}{|c|}{ Televisión: Televisora local, TVSultana Canal 13.} \\
\hline Alcance & \multicolumn{3}{|c|}{ Clientes reales y potenciales. } \\
\hline \multirow[t]{2}{*}{ Costo } & Diario & Semanal & Mensual \\
\hline & $\$ 50,00$ & $\$ 100,00$ & $\$ 400,00$ \\
\hline
\end{tabular}

La televisión es un medio masivo que logra una difusión mucho más efectiva que la radio, puesta que genera un mayor impacto a través de los soportes televisivos. En cuanto a la radio, es un buen canal de marketing promocional y una de las estrategias más utilizadas para difundir cualquier información gracias al gran impacto que genera 
Table 12

Estrategia 3. Cortinas LED.

\begin{tabular}{|c|c|c|c|}
\hline Descripción & \multicolumn{3}{|c|}{$\begin{array}{l}\text { Las cortinas LED situados en las zonas de mayor concurrencia de personas } \\
\text { permitirán brindar mayor información sobre la marca y los productos que oferta. }\end{array}$} \\
\hline Objetivo & \multicolumn{3}{|c|}{$\begin{array}{l}\text { Captar la atención del público de una manera atractiva, dinámica y en tiempo } \\
\text { real. }\end{array}$} \\
\hline Estrategia & \multicolumn{3}{|c|}{ Ubicar cortinas LED en las zonas estratégicas de la ciudad. } \\
\hline Audiencia a impactar & \multicolumn{3}{|c|}{ Clientes reales y potenciales. } \\
\hline Responsable & \multicolumn{3}{|c|}{ Departamento de Marketing. } \\
\hline Medio & \multicolumn{3}{|l|}{ Visual. } \\
\hline Táctica & \multicolumn{3}{|c|}{$\begin{array}{l}\text { - Utilizar contenidos precisos que capte la atención de los transeúntes, según e } \\
\text { sitio estratégico fijado (parroquia Veloz). }\end{array}$} \\
\hline Frecuencia & \multicolumn{3}{|l|}{ Anual. } \\
\hline \multirow[t]{2}{*}{ Costo } & Cantidad & Costo unitario & Costo total \\
\hline & 1 & $\$ 800,00$ & $\$ 800,00$ \\
\hline
\end{tabular}

en el público. Con el fin de acceder a un público más amplio y lograr que la audiencia se incline hacia la compra del producto en nuestra empresa.

\subsection{Medios de comunicación BTL}

Table 13

Estrategia 4. Material P.O.P.

\begin{tabular}{|c|c|c|c|}
\hline Descripción & \multicolumn{3}{|c|}{$\begin{array}{l}\text { El material P.O.P que recurre a la publicidad puesta en los puntos de venta, } \\
\text { busca generar una permanencia de la marca recurriendo a una gran variedad } \\
\text { de objetos donde se puede imprimir o estampar información de la empresa o } \\
\text { producto. }\end{array}$} \\
\hline Objetivo & \multicolumn{3}{|c|}{ Impulsar la compra y generar impacto de los productos en el punto de venta. } \\
\hline Estrategia & \multicolumn{3}{|c|}{$\begin{array}{l}\text { Imprimir material P.O.P con información relevante de la empresa y los productos } \\
\text { que oferta. }\end{array}$} \\
\hline Audiencia a impactar & \multicolumn{3}{|c|}{ Clientes reales y potenciales. } \\
\hline Responsable & \multicolumn{3}{|c|}{ Departamento de Marketing. } \\
\hline Medio & \multicolumn{3}{|c|}{ Impresos: agencia publicitaria 'Orange’. } \\
\hline Táctica & \multicolumn{3}{|c|}{$\begin{array}{l}\text { Colocar habladores en el punto de venta. Diseñar afiches y repartirlos en las } \\
\text { zonas más transitadas de la parroquia Velasco (CC. Multiplaza) y Lizarzaburu } \\
\text { (CC. La Condamine). }\end{array}$} \\
\hline Frecuencia & \multicolumn{3}{|l|}{ Mensual } \\
\hline \multirow[t]{2}{*}{ Costo } & Cantidad & Costo unitario & Costo total \\
\hline & 100 & $\$ 0,20$ & $\$ 20,00$ \\
\hline
\end{tabular}

El material P.O.P (Point of Purchase) que recurre a la publicidad puesta en los puntos de venta, busca generar una permanencia de la marca recurriendo a una gran variedad de objetos donde se puede imprimir o estampar información de la empresa o producto. 


\section{Table 14}

Estrategia 5. Vallas publicitarias.

\begin{tabular}{|c|c|c|c|c|}
\hline Descripción & \multicolumn{4}{|c|}{$\begin{array}{l}\text { Las vallas publicitarias son útiles cuando se desea que el negocio sea más visible al } \\
\text { público, ya que se encuentra exhibido las } 24 \mathrm{hr} \text { del día los } 365 \text { días del año. }\end{array}$} \\
\hline Objetivo & \multicolumn{4}{|c|}{$\begin{array}{l}\text { Generar un impacto visual que persuada el consumo de los productos que posicione } \\
\text { la marca. }\end{array}$} \\
\hline Estrategia & \multicolumn{4}{|c|}{$\begin{array}{l}\text { Captar la atención de las personas a todas horas, mientras la persona va caminando } \\
\text { o manejando el coche. }\end{array}$} \\
\hline $\begin{array}{l}\text { Audiencia a } \\
\text { impactar }\end{array}$ & \multicolumn{4}{|c|}{ Clientes reales y potenciales. } \\
\hline Responsable & \multicolumn{4}{|c|}{ Departamento de Marketing. } \\
\hline Medio & \multicolumn{4}{|l|}{ Impreso. } \\
\hline Táctica & \multicolumn{4}{|c|}{$\begin{array}{l}\text { - Contratar un espacio para brindar información sobre la empresa mediante vallas } \\
\text { publicitarias. } \\
\text { - Colocar las vallas en las zonas más transitadas de la ciudad (Bypass, Terminal } \\
\text { terrestre). }\end{array}$} \\
\hline Frecuencia & \multicolumn{4}{|l|}{ Anual. } \\
\hline Alcance & \multicolumn{4}{|c|}{ Clientes reales y potenciales. } \\
\hline \multirow[t]{2}{*}{ Costo } & Cantidad & $\begin{array}{l}\text { Costo unitario } \\
\text { impreso }\end{array}$ & $\begin{array}{l}\text { Costo alquiler de } \\
\text { estructura }\end{array}$ & Costo total \\
\hline & 2 & $\$ 15,00$ & $\$ 50,00$ & $\$ 130,00$ \\
\hline
\end{tabular}

El objetivo será Impulsar la compra y generar impacto de los productos en el punto de venta.

\subsection{Medios digitales: Redes sociales y pagina web}

Las redes sociales hoy en día es el medio más utilizado por la mayoría de las personas desde adolescentes hasta personas de tercera edad; así mismo la página web representa un medio por el cual el cliente obtiene mayor información de forma rápida en cualquier parte del mundo, aparte que permite la compra en línea. Con el fin de generar una interacción entre el cliente y la marca, a la vez incentivar la compra en línea.

\section{Conclusiones}

El Supermercado Total Home para posicionarse en el mercado riobambeño, debe manejar una comunicación efectiva que contribuya a que la marca logre posicionarse en la mente del consumidor; los resultados de la investigación de mercado determinaron que la empresa maneja una nula comunicación con el público externo, tampoco existe difusión de la marca ni de los productos que ofrece, todo esto debido a que la empresa cuenta con poca experiencia por los años que lleva dentro del mercado apenas 3 
Table 15

Estrategia 6. Creación de redes sociales.

\begin{tabular}{|c|c|c|c|}
\hline Descripción & \multicolumn{3}{|c|}{$\begin{array}{l}\text { Las redes sociales hoy en día son el medio más utilizado por la mayoría de las } \\
\text { personas en todas las edades. }\end{array}$} \\
\hline Objetivo & \multicolumn{3}{|c|}{ Captar nuevos seguidores y visitas en redes sociales. } \\
\hline Estrategia & \multicolumn{3}{|c|}{$\begin{array}{l}\text { Generar publicaciones pagadas en Facebook, dando a conocer promociones, } \\
\text { ofertas, sorteos, con la finalidad de logra interacción entre el cliente y la marca. }\end{array}$} \\
\hline Audiencia a alcanzar & \multicolumn{3}{|c|}{ Clientes reales y potenciales. } \\
\hline Responsable & \multicolumn{3}{|c|}{ Departamento de Marketing. } \\
\hline Medio & \multicolumn{3}{|l|}{ Virtual. } \\
\hline Táctica & \multicolumn{3}{|c|}{$\begin{array}{l}\text { - Realizar sorteos en temporadas especiales. } \\
\text { - Ofrecer premios sorpresa al usuario con más 'likes' o comentarios en nuestra } \\
\text { publicación. } \\
\text {-Publicitar contenidos de mayor interés para el consumidor, como por ejemplo } \\
\text { las ofertas de la semana, promociones, descuentos, etc. }\end{array}$} \\
\hline Frecuencia & \multicolumn{3}{|l|}{ Diario. } \\
\hline \multirow[t]{2}{*}{ Costo } & Semanal & Mensual & Total anual \\
\hline & $\$ 5,00$ & $\$ 20,00$ & $\$ 240,00$ \\
\hline
\end{tabular}

Table 16

Estrategia 6. Página web empresarial.

\begin{tabular}{|c|c|c|c|}
\hline Objetivo & \multicolumn{3}{|c|}{$\begin{array}{l}\text { Motivar la compra en línea de los productos con total confiabilidad, de manera } \\
\text { rápida y sencilla. }\end{array}$} \\
\hline Estrategia & \multicolumn{3}{|c|}{ Crear una página web en la que los clientes puedan realizar su compra en línea. } \\
\hline Audiencia a alcanzar & \multicolumn{3}{|c|}{ Clientes reales y potenciales. } \\
\hline Responsable & \multicolumn{3}{|c|}{ Departamento de Marketing. } \\
\hline Medio & \multicolumn{3}{|l|}{ Virtual. } \\
\hline Táctica & \multicolumn{3}{|c|}{$\begin{array}{l}\text { Diseñar una página web que permita al usuario realizar la compra en línea y } \\
\text { obtener mayor información. }\end{array}$} \\
\hline Frecuencia & \multicolumn{3}{|l|}{ Anual. } \\
\hline \multirow[t]{2}{*}{ Costo } & Cantidad & Costo unitario & Costo total \\
\hline & 1 & $\$ 500$ & $\$ 500,00$ \\
\hline
\end{tabular}

años y por tanto no ha logrado establecer estrategias de comunicación que permitan el cumplimiento de objetivos.

La ausencia de un plan de comunicación en las organizaciones constituye un grave error que puede llevar al fracaso de la empresa, debido a que la misma puede constituirse en una hoja de ruta que sirva de guía para la consecución de objetivos, a la vez que incluya actividades y procedimientos en un periodo determinado con la finalidad de proyectar una buena imagen de la empresa hacia el público objetivo.

Las estrategias de comunicación se basan en los resultados obtenidos en el estudio de mercado; debido a que a través de los datos que arroja el mismo se puede conocer la percepción del consumidor y frente a ello responder y cumplir con las expectativas del 
cliente, tomando en cuenta que el mercado hoy en día es cambiante y requiere mayor exigencia. Cabe mencionar que las estrategias más efectivas a considerar son aquellas que parten del análisis del FODA de la empresa, específicamente de las debilidades, en el caso del Supermercado Total Home un factor clave es el débil posicionamiento que posee en el mercado, en congruencia, la mejor opción será implementar estrategias de comunicación mediante herramientas efectivas en los medios de mayor aceptación tal como lo señala la Tabla 6 .

Mediante el estudio de mercado realizado y el análisis de factores internos y externos, se evidenció que la empresa no aplica estrategias que permitan posicionar la marca entre las más destacadas de la ciudad y mucho menos de la provincia; dado al desconocimiento que poseen los directivos sobre dicho tema y la poca experiencia en el sector. Por lo tanto, los resultados expuestos en la Tabla 5, en donde el $90 \%$ de las personas encuestadas afirman que si les gustaría recibir más información sobre la empresa, la empresa cuenta con una excelente oportunidad para establecer estrategias de comunicación que contribuyan con el posicionamiento de la misma.

\section{References}

[1] Wolton D. Pensar la comunicación. Buenos Aires: Prometeo Libros; 2007.

[2] Alard J, Monfort A. Plan de comunicación on y off en la práctica. Madrid: ESIC; 2017.

[3] Quijada V. Comunicación oral y escrita. México: UNID; 2014.

[4] Ferraretto R. Plan de Comunicación, herramienta de las relaciones públicas. Madrid: EAE; 2011.

[5] Valero M. Ejemplo de plan de comunicación. Milejemplos. Disponible en: https: //www.milejemplos.com/empresa/ejemplo-de-plan-de-comunicacion.html?utm source=emprendepyme.net\&utm_medium=Network\&utm_campaign=post_button

[6] Guijarro M. Comunicación Digital. IEBSchool. Disponible en: https://www.iebschool. com/blog/elaborar-plan-comunicacion-9-pasos-comunicacion-digital/

[7] Quero J. Cómo elaborar un plan de comunicación. Raiolanetworks. Disponible en: https://raiolanetworks.es/blog/plan-de-comunicacion/

[8] Juliá J. Posicionarse o desaparecer. Madrid: ESIC; 2015.

[9] Luna AC. Posicionamiento we (Seo/Sem). Málaga: ICB Editores; 2017.

[10] Leyva A. Marketing en esencia: Gestionar tu marca personal, profesional y empresarial. Buenos Aires: Granica; 2016.

[11] González N. Posicionamiento de marca frente a una identidad de marca. Magentaig. Disponible en: https://magentaig.com/ posicionamiento-de-marca-frente-a-identidad-de-marca-cual-es-la-difere.

[12] Ruiz J. Metodología de la investigación Cualitativa. Bilbao: DEUSTO; 2012. 
[13] Gómez M. Introducción a la metodología de la Investigación científica. Córdoba: Brujas; 2006.

[14] Naghi M. Metodología de la Investigación. México: Limusa; 2000.

[15] Rodríuez E. Metodología de la Investigación. México: Univdersidad Juárez Autónoma de Tabasco; 2005

[16] Trenza A. Análisis PESTEL: Qué es y para qué sirve. Anatrenza. Disponible en: https://anatrenza.com/analisis-pestel/

[17] Riqueleme M. La matriz del perfil competitivo. Webyempresas. 2015 May 24. Disponible en de https://www.webyempresas.com/la-matriz-del-perfil-competitivo/

[18] Galiana P. Descubre las 5 fuerzas de Porter y cómo aprovehcarlas. IEBSchool. 2016 December 9. Disponible en://www.iebschool.com/blog/ las-5-fuerzas-porter-marketing-digital/

[19] Min SY. Matriz de evaluación de factores externos. Yiminshum. 2018 June 18. Disponible en: https://yiminshum.com/ matriz-evaluacion-factores-externos-matriz-efe-mefe/

[20] Espinosa R. ¿Qué es el modelo AIDA en marketing? Robertoesponosa. 2017 April 11. Disponible en: https://robertoespinosa.es/2017/04/11/ modelo-aida-marketing-metodo

[21] Fernández F. Comunicación efectiva y trabajo en equipo. Logroño: Tutor Formación; 2016. 